\title{
Probiotics Based Approaches to Target Mucosal Compartments: Potential Novel Approaches to HIV Vaccine Development
}

Venkateswarlu Chamcha*

Emory Vaccine Center, Yerkes National Primate Research Center, Emory University, Atlanta, Georgia, USA

It's been 35 years since the first cases of AIDS were reported. Since then, we have witnessed tremendous advances in prevention, testing, and treatment. Although, biomedical interventions to reduce HIV-1 acquisition have favorably influenced the trajectory of HIV1 infections in several populations throughout the worldwide, the key to ending the HIV epidemic is a protective, affordable vaccine that prevents the virus from establishing infection and the need for a globally effective HIV-1 prophylactic vaccine is more compelling than ever. The RV144 study using a poxvirus vector prime and envelope protein boost strategy demonstrated a modest but statistically significant level of efficacy and established the concept that a vaccine could prevent HIV infection [1]. The beauty of a vaccine is that once a person has been vaccinated, the body automatically responds to protect itself against infection- it does not require a conscious action on the part of the potentially exposed person. Indeed, history teaches us that a protective vaccine, as opposed to treatment alone, is the only way to eradicate a pathogen-the vaccines for polio or smallpox is great examples of this. Because HIV attacks and can lay dormant in cells of the immune system, developing a vaccine against it is one of the more difficult scientific problems of our time. Progress is being made, and many vaccine concepts or approaches to boost the durability and efficacy of HIV vaccines underdevelopment.

\section{Presence of Vaccine-specific Responses at Mucosal Compartments Enhances the Protection}

Mucosal tissues mark the boundary between the environment and the body's interior and several layers of constitutive defensive barriers confront any virus that seeks to enter the host through mucosal surfaces. HIV enters through the mucosal surfaces by infecting the CD4+ T cells present at these tissue surfaces initially and enters into the gastro-intestinal (GI) tract where it replicates many folds before it disseminate into multiple systemic compartments. Neutralizing Abs (nAbs), so called because they neutralize the biological effects of an antigen, inhibit viral attachment or entry. nAbs that are secreted into the mucosal lumen can bind to an incoming pathogen and prevent it from entering host target cells by blocking receptor binding or cellular uptake. Complement fixation by $\mathrm{nAb}$ kills viruses. Moreover, Envelopespecific, nAbs provide sterilizing immunity at little cost to the host. Apart from nAbs, as evidenced clearly, at least in non-human primate (NHP) studies, the strong avidity Envelope binding, non-neutralizing antibodies (nnAb), present in the rectal secretions, have showed an important direct correlations to the protection potentially through antibody dependent cell mediated cytotoxicity (ADCC) [2]. Finally, vaccines eliciting high titers of $\mathrm{nAb}$, or high avidity nnAbs in mucosal tissue compartments are thus ideal for providing protective immunity against HIV-1.

\section{Immunizations Using Live Probiotic Organisms are Suitable to Deliver Antigens that Generate Antibodies at Mucosal Compartments}

Generally, immunization through the mucosal route has elicited far better responses in mucosal tissue than immunization by systemic routes.
For example, the oral route of immunization is the best way to induce a strong immunity in the gut [3]. However, most of the HIV vaccine regimens that are being evaluated in humans are not administered through mucosal routes (oral, vaginal or rectal) because either they don't withstand the hostile acidic environment in the stomach when delivered orally or it is hard to escape the activity of oral proteases or the vaginal and rectal routes are not practical to use. Furthermore, a specific feature of HIV infection is the rapid depletion of CD4 T cells in the gut within days after infection and this happens irrespective of the route of infection [4]. This early depletion is not reversible following antiretroviral therapy and contributes to rapid disease progression. Thus, there is a great need for the development of an HIV vaccine that can be delivered orally and is capable of inducing potent anti-viral immunity in the gut with the potential to block or control HIV replication and prevent infection and/or rapid loss of CD4 T cells.

The gastro-intestinal (GI) tract is heavily colonized by bacteria and some of these or their derivatives can protect the host against viral infections. If HIV antigen/s are delivered successfully to GI tracts through a probiotic/s, and stimulate immune responses at these compartments, it can serve as a great toll to deliver HIV antigens or immune stimulatory molecules (adjuvants) directly to the GI tract. Many research groups have tried to target the GI tract by delivering $\mathrm{HIV}$ antigens using various probiotic organisms. However, there is very limited success achieved in this front. Recently, in a proof of concept study, a live probiotic organism, Lactococcus lactis (L. lactis), that transiently present in the human small intestine, have been successfully utilized to deliver HIV-1 Gag antigen [5]. Lactococcus species have been explored as vaccine vectors for generating mucosal immunity against infectious diseases. The key advantages of using a Lactococcus vaccine vector/s are: 1) Lactococcus is a GRAS (Generally Regarded As Safe) organism, 2) it naturally withstands stomach acids and bile, 3) it can be administered repeatedly since it survives only temporarily in the intestinal tract and does not colonize humans, 4) it has intrinsic adjuvant properties, 5) it does not require a cold chain, and 6) it is inexpensive to produce. Also Lactococcus is a Gram-positive bacterium and therefore does not possess endotoxic lipopolysaccharides (LPS), which are associated with commonly used vaccine strain Gram-negative bacteria such as Escherichia coli and Salmonella typhimurium.

*Corresponding author: Dr. Venkateswarlu Chamcha, Ph.D., Department of Microbiology and Immunology, Emory Vaccine Center, Yerkes National Primate Research Center, Emory University, Atlanta, Georgia, USA 30329; Tel: 404727 1432; Fax: 404727 7768; E-mail: vchamch@emory.edu

Received May 24, 2016; Accepted May 26, 2016; Published May 31, 2016

Citation: Chamcha V (2016) Probiotics Based Approaches to Target Mucosa Compartments: Potential Novel Approaches to HIV Vaccine Development. J AIDS Clin Res 7: e119. doi:10.4172/2155-6113.1000e119

Copyright: (c) 2016 Chamcha V. This is an open-access article distributed under the terms of the Creative Commons Attribution License, which permits unrestricted use, distribution, and reproduction in any medium, provided the original author and source are credited. 


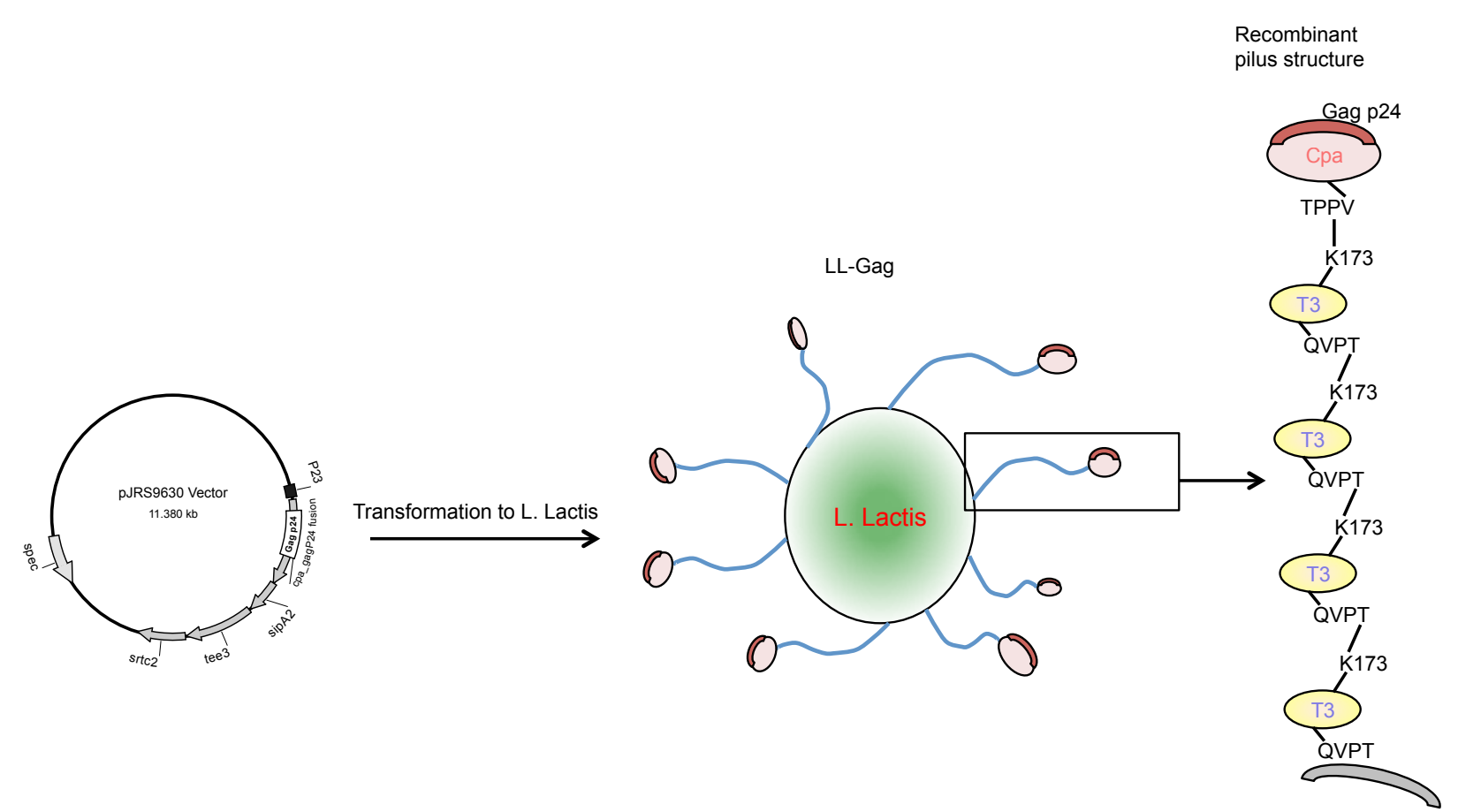

Figure 1: Schematic of making LL-Gag. The pJRS930 vector that carries the HIV-1 Gag p24 gene and other genes that are necessary for making or polymerizing pilus were transformed into Lactococcus lactis bacterial cells which intern express these in concert to make a pilus that present Gag p24 protein at the tip of it. We call this bacteria as LL-Gag.

\section{Presentation of Antigen on Tip of the Pilus is Key to the Success of Immunization Using L. lactis}

Earlier vaccine studies using $L$. lactis or other probiotics have been tested by engineering the organism to present antigen/s either on their surfaces or secreted out into the GI environment. Such approaches have achieved a very limited success in generating antigen-specific responses mainly due to the poor antigen uptake and presentation by the host's immune cells (DCs, B cells, etc.). This can be overcome by using an UPTOP (Unhindered Presentation on Tips of Pili) system for the expression of the HIV Gag protein on the lactococcal surface (Figure 1). UPTOP utilizes the T3 pilus of Streptococcus pyogenes (the group A streptococcus or GAS) to present a desired antigen to the immune system. By engineering a plasmid encoding the GAS T3 pilus with cpa fused to gag P24 an antigen from an HIV-1 clade B, authors have expressed it in L. lactis strain MG1363 (called as LL-Gag). They then intra-gastrically immunized BALB/c mice with LL-Gag and observed potent humoral and cellular immune responses in mucosal and systemic compartments. Interestingly, when they compared the LL-Gag induced gut-associated immune responses to those of an intramuscular DNA primed and MVA boosted vaccine modality, which is in clinical studies in the USA, it demonstrated that LL-Gag induced stronger humoral immunity in the gut. Finally, they demonstrated a critical role for the GAS pilus in the activation of a specific subset (CD103+ or CD11b+) of mucosal dendritic cells that may be important for the immunogenicity of Gag. These results demonstrated that oral administration of probiotic L. lactis expressing the antigen on the tip of the GAS pilus induces strong mucosal IgG and $\operatorname{IgA}$ antibody responses in the gut of mice. Together their results reveal a promising novel immunization strategy to elicit strong humoral and cellular mucosal immunity against HIV. Furthermore, these approaches prove great promise and may be tested in other vaccine development approaches against various enteric viral diseases.

\section{References}

1. Rerks-Ngarm S, Pitisuttithum $P$, Nitayaphan $S$, Kaewkungwal J, Chiu J, et al (2009) Vaccination with ALVAC and AIDSVAX to prevent HIV-1 infection in Thailand. New Engl J Med 361: 2209-2220.

2. Chamcha V, Kannanganat S, Gangadhara S, Nabi R, Kozlowski PA, et al (2016) Strong, but age-dependent, protection elicited by a deoxyribonucleic acid/modified vaccinia ankara simian immunodeficiency virus vaccine. Open Forum Infect Dis 3: ofw034.

3. Zhou Q, Hidajat R, Peng B, Venzon D, Aldrich MK, et al. (2007) Comparative evaluation of oral and intranasal priming with replication-competent adenovirus 5 host range mutant (Ad5hr)-simian immunodeficiency virus (SIV) recombinant vaccines on immunogenicity and protective efficacy against SIVmac251. Vaccine 25: 8021-8035.

4. Mattapallil JJ, Douek DC, Hill B, Nishimura Y, Martin M, et al. (2005) Massive infection and loss of memory CD4(+) T cells in multiple tissues during acute SIV infection. Nature 434: 1093-1097.

5. Chamcha V, Jones A, Quigley BR, Scott JR, Amara RR (2015) Ora Immunization with a recombinant Lactococcus lactis-expressing HIV-1 antigen on Group A Streptococcus pilus Induces strong mucosal immunity in the Gut. J Immunol 195: 5025-5034. 